\title{
Influência da mídia televisiva no consumo de bebidas alcoólicas por universitários
}

\author{
Alana Oliveira Porto ${ }^{1}$ \\ Marcela Andrade Rios ${ }^{1}$ \\ Dieslley Amorim de Souza ${ }^{1}$
}

\begin{abstract}
Objetivou-se discutir a publicidade de bebidas alcoólicas na mídia televisiva e o consumo de bebidas alcoólicas por universitários por meio de uma revisão integrativa de literatura. $\mathrm{O}$ estudo foi realizado com 25 artigos, das bases de dados LILACS e MEDLINE, publicados entre os anos 2011 e 2017 . Verificou-se que a referência ao consumo de álcool na televisão tem atingido grupos vulneráveis utilizando-se de diversas estratégias que incentivam este consumo. Considera-se necessário educar os jovens de maneira que estes exerçam sua criticidade na análise do que é exposto pela mídia.
\end{abstract}

Descritores: Consumo de Bebidas Alcoólicas; Propaganda; Universidade.

${ }^{1}$ Universidade do Estado da Bahia, Guanambi, BA, Brasil. 


\title{
Influence of television media on alcohol consumption
}

\author{
by university students
}

The objective was to discuss the advertising of alcoholic beverages in the television media and the consumption of alcoholic drinks by university students, through an integrative literature review. The study was conducted with 25 articles from the LILACS and MEDLINE databases, published between 2011 and 2017. It has been verified that the reference to alcohol consumption on television has reached vulnerable groups, using several strategies that encourage this consumption. It is considered necessary to educate young people so that they exercise their criticality in the analysis of what is exposed by the media.

Descriptors: Consumption of Alcoholic Beverages; Advertising; University.

\section{Influencia de los medios televisivos en el consumo de bebidas alcohólicas por universitários}

\begin{abstract}
Se objetivó discutir la publicidad de bebidas alcohólicas en los medios televisivos y el consumo de bebidas alcohólicas por universitarios, a través de una revisión integrativa de literatura. EI estudio fue realizado con 25 artículos de las bases de datos LILACS y MEDLINE, publicados entre los años 2011 y 2017. Se verificó que la referencia al consumo de alcohol en la televisión ha alcanzado a grupos vulnerables, utilizando de diversas estrategias que incentivan este consumo. Se considera necesario educar a los jóvenes de manera que éstos ejerzan su criticidad en el análisis de lo que es expuesto por los medios.
\end{abstract}

Descriptores: Consumo de Alcohol; Publicidad; Universidad.

\section{Introdução}

O consumo de bebidas alcoólicas é um comportamento de risco à saúde responsável por cerca de 2,5 milhões de mortes por ano. No entanto, este produto é bem aceito socialmente representando a substância psicoativa mais consumida no mundo(1).

Apesar de ser uma droga lícita, o álcool tem potencial de causar diversas consequências à sociedade, como a ocorrência de acidentes de trânsito, agressões, relações sexuais desprotegidas, doenças mentais e morte. Mesmo diante de tantos agravos, seu consumo continua sendo indiscriminado e está em ascensão nas faixas etárias mais jovens ${ }^{(2)}$.

Os universitários apresentam elevada prevalência no consumo de bebidas alcoólicas ${ }^{(3)}$ e esse consumo está pautado na idealização da socialização e, posteriormente, na autoafirmação de sua independência visto que, nessa fase, esses jovens experimentam um momento de autonomia e, consequentemente, tornamse mais vulneráveis ao consumo de álcool(4).

Dentre as bebidas alcoólicas, a cerveja destaca-se como a mais consumida e seu uso está associado à possibilidade de relaxamento, prazer, desinibição e à idealização da facilidade de socialização e construção de vínculos ${ }^{(3)}$. Essas convicções referentes à ingestão de cerveja são fortemente evocadas pela mídia, por meio dos artifícios de marketing televisivo, induzindo o consumidor à associação do consumo de álcool aos momentos divertidos e fantasiando, assim, uma pseudorrealidade $e^{(2,5)}$.

Embora a discussão acerca das propagandas de bebidas alcoólicas não seja tão recente, poucas são as políticas de prevenção de consumo e restrição do marketing de bebidas alcoólicas ${ }^{(5)}$. Segundo a Lei no 9.294 , de 15 de julho de 1996, é vetada a vinculação do consumo de bebidas alcoólicas com esportes, saúde, automóveis e êxito sexual, bem como fica imposta a obrigatoriedade da utilização de advertências do tipo "Beba com moderação", 
"Produto destinado a adultos" ou nos anúncios comerciais. Porém, existe o entendimento de que a bebida alcoólica é apenas a que apresenta teor alcoólico maior que 13 graus. Gay-Lussac (GL), neste sentido, afirma que a cerveja fica excluída de tais restrições visto que ela possui GL consideravelmente inferior(6).

A fiscalização do cumprimento de tal lei fica a cargo do Conselho de Autorregulação Publicitária (CONAR), que é um órgão não governamental e apresenta, como estratégia, a teoria da autorregulamentação, que se refere ao controle do conteúdo das propagandas pautado na ética das empresas. No entanto, existem diversas violações às leis de restrição fazendo com que ocorra a exposição do público vulnerável ao consumo de bebidas alcoólicas ${ }^{(5)}$.

Ao considerar a ascensão do consumo de bebidas alcoólicas por jovens no meio universitário e suas consequências, este estudo tem por objetivo discutir a publicidade de bebidas alcoólicas na mídia televisiva e o consumo de bebidas alcoólicas por universitários a partir da literatura científica.

\section{Metodologia}

Trata-se de uma revisão integrativa da literatura que busca identificar, nas publicações científicas, o que há disponível sobre a influência da mídia televisiva no consumo de bebidas alcoólicas por universitários. Para tanto, foram seguidas as seis etapas: estabelecimento do objetivo; estabelecimento de critérios de inclusão e exclusão de artigos (seleção da amostra); definição das informações a serem extraídas dos artigos selecionados; análise dos resultados; discussão e apresentação dos resultados. A última etapa consistiu na apresentação da revisão( ${ }^{(7)}$.

A pergunta norteadora, para a elaboração da revisão integrativa, foi construída a partir da estratégia PICO $(\mathrm{P}=$ population, $\mathrm{I}=$ intervention, $\mathrm{C}=$ control, $\mathrm{O}=$ outcomes $)$ e consistiu em: Quais são as influências exercidas pela mídia no consumo de bebidas alcoólicas por universitários?

Para o desenvolvimento do estudo, foram realizadas buscas eletrônicas na Biblioteca Virtual em Saúde (BVS), nas bases de dados Literatura Latino-Americana e do Caribe em Ciências da Saúde (LILACS), Medical Literature Analysis and Retrieval System Online (MEDLINE) e BDENF utilizando-se os Descritores em Ciências da Saúde (DEC's) "bebidas alcoólicas", "televisão", "propaganda" e "universidade" inter-relacionados com o operador booleano AND.

Os critérios de inclusão foram artigos completos, disponíveis e indexados, publicados no período de 2011 a 2017 e disponíveis nas línguas portuguesa e inglesa.

$\mathrm{Na}$ primeira busca, associaram-se os descritores "bebidas alcoólicas" AND "televisão" resultando em
234 publicações. Após passarem pelos critérios de inclusão, restaram 51 estudos, porém, depois da leitura dos resumos, esse número decresceu para 11.

Na segunda busca, foram associados os descritores "bebidas alcoólicas" AND "propaganda" resultando em 44 publicações. Após passarem pelos critérios de inclusão, restaram cinco artigos, porém, depois da leitura dos resumos, esse número decresceu para dois.

$\mathrm{Na}$ terceira busca, associaram-se "bebidas alcoólicas" AND "universidade", que resultou em 2.022 publicações. Após passarem pelos critérios de inclusão, restaram 44 estudos, porém, depois da leitura dos resumos, esse número decresceu para 12.

Ao final das buscas, elencaram-se 25 artigos (Figura 1) lidos na íntegra a fim de permitir a análise e a classificação temática do conteúdo.

Elaborou-se um instrumento para a coleta dos dados contemplando informações relacionadas à identificação do artigo, ao tipo da publicação, às características metodológicas, aos principais achados e à classificação do nível de evidência.

Para a classificação quanto ao poder de evidência, utilizou-se a Classificação Norte-Americana Hierárquica de Evidências: nível 1 - metanálise de múltiplos estudos controlados; nível 2 - estudo individual com delineamento experimental; nível 3 - estudo com delineamento quaseexperimental como estudo sem randomização com grupo único pré e pós-teste, séries temporais ou caso-controle; nível 4 - estudo com delineamento não experimental como pesquisa descritiva correlacional e qualitativa ou estudos de caso; nível 5 - relatório de casos ou dado obtido de forma sistemática, de qualidade verificável ou dados de avaliação de programas; nível 6 - opinião de autoridades respeitáveis baseada na competência clínica ou opinião de comitês de especialistas incluindo interpretações das informações não baseadas em pesquisas ${ }^{(7)}$.

A síntese dos dados extraídos apresenta-se de forma descritiva contemplando a quinta e a sexta etapas da revisão integrativa. Por meio da Análise Temática ou Categorial, tipo de técnica de Análise de Conteúdo, operouse o desmembramento do texto em unidades (categorias) segundo reagrupamentos sistemáticos analógicos.

A análise constitui-se pela leitura dos vinte e cinco artigos selecionados ${ }^{(8)}$. Posteriormente, buscouse descobrir os núcleos de sentido que compõem o corpus do estudo preocupando-se com a frequência desses núcleos, sob a forma de dados segmentáveis e análogos, onde se realizou nova análise e, dela, emergiram três categorias, respectivamente: Fatores determinantes do consumo de bebidas alcoólicas por universitários, Políticas de restrição publicitária de bebidas alcoólicas e Estratégias de marketing das empresas de bebidas alcoólicas. 


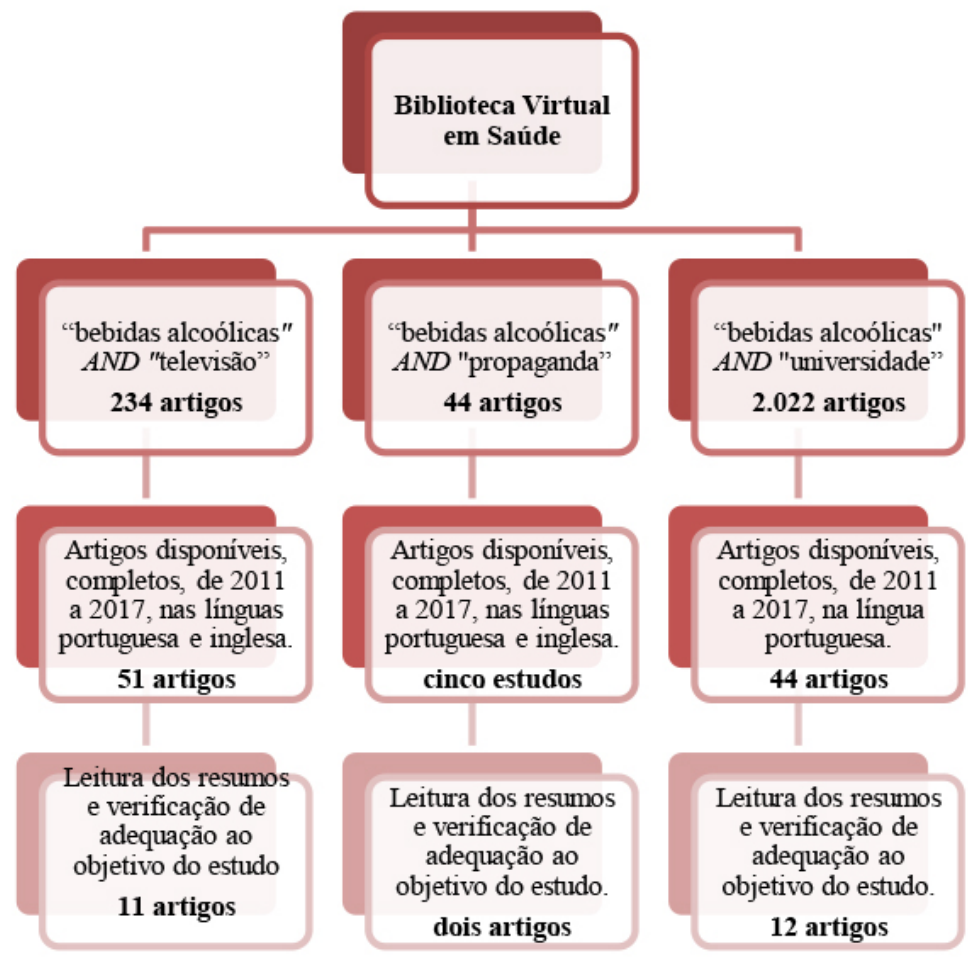

Figura 1 - Busca e seleção das publicações utilizadas para a construção deste estudo

\section{Resultados}

Para a revisão integrativa, analisaram-se 25 artigos lidos na íntegra e que atenderam aos critérios de inclusão e ao objetivo previamente estabelecido. Dentre os artigos encontrados, a diferença de distribuição nas bases de dados foi mínima, sendo 13 (52\%) disponíveis na LILACS e 12 (48\%) disponíveis na MEDLINE. Em relação ao ano, o maior quantitativo de publicações ocorreu no ano de 2014 (24\%) seguido de 2012 e 2015, com $20 \%$ em ambos, 2013 e 2016 , com $12 \%$ em ambos, 2011 (8\%) e 2017 (4\%).

Todos os estudos estavam vinculados a alguma instituição de ensino superior, sendo que quatro (16\%) procederam da Universidade Federal de São Paulo; dois (8\%), da Universidade de Boston; dois (8\%), da Escola de Saúde Pública Johns Hopkins Bloomberg; dois (8\%), da Universidade Federal do Rio Grande do Sul; dois (8\%), da Universidade Estadual do Rio de Janeiro; um (4\%), da Universidade de Newcastle; um (4\%), da Universidade de Nottingham; um (4\%), da Universidade de Akron; um (4\%), da Universidade de Manchester; um (4\%), da Faculdade de Medicina de Dartmouth; um (4\%), da Universidade Estadual de Campinas; um (4\%), da Universidade Estadual do Ceará; um (4\%), da Universidade Estadual de Montes Claros; um (4\%), da Universidade Federal de Minas Gerais; um (4\%), do Instituto Nacional de Câncer José Alencar Gomes da Silva; um (4\%), da Universidade Deakin; um (4\%), do Norris Cotton Cancer Center e um (4\%) procedeu da Universidade Federal de Alfenas.

A relação dos estudos internacionais e nacionais selecionados para a construção desta revisão encontrase disponível nas figuras 2 e 3 , respectivamente.

Como apontam os dados acima, houve estudos de diversas nacionalidades e são nítidas as diferentes vertentes, pois, dos estudos internacionais, 11 (44\%) trazem um enfoque para a relação mídia televisiva versus consumo de bebidas alcoólicas, como pode ser visto na figura 2. Nos estudos nacionais, 14 (56\%), nota-se que a principal discussão é voltada para o consumo de álcool no meio universitário, pois apenas dois estudos $(14,2 \%)$ discutem a relação mídia televisiva versus consumo de bebidas alcoólicas, como retrata a figura 3.

\begin{tabular}{|l|l|l|}
\hline Título/Autor/Ano & $\begin{array}{l}\text { Tipo de Estudo/ } \\
\text { Nível de Evidência }\end{array}$ & Objetivos \\
\hline $\begin{array}{l}\text { Alcohol marketing in televised international } \\
\text { football: frequency analysis/Adams et al., } 2014\end{array}$ & Transversal/Nível IV & $\begin{array}{l}\text { Determinar a frequência das referências visuais para } \\
\text { o álcool em partidas EURO2012 no Reino Unido. }\end{array}$ \\
\hline $\begin{array}{l}\text { Alcohol imagery on popularly viewed } \\
\text { television in the UK/Lyons et al., } 2013\end{array}$ & Transversal/Nível IV & $\begin{array}{l}\text { Medir a ocorrência de imagens de álcool } \\
\text { transmitidas pela televisão em horário nobre no } \\
\text { Reino Unido. }\end{array}$ \\
\hline
\end{tabular}




\begin{tabular}{|c|c|c|}
\hline Título/Autor/Ano & $\begin{array}{l}\text { Tipo de Estudo/ } \\
\text { Nível de Evidência }\end{array}$ & Objetivos \\
\hline $\begin{array}{l}\text { Alcohol Advertising in Sport and Non-Sport } \\
\text { TV in Australia, during Children's Viewing } \\
\text { Times / O'Brien et al., } 2015\end{array}$ & Transversal/Nível IV & $\begin{array}{l}\text { Estimar a quantidade de publicidade de álcool em } \\
\text { canais esportivos e não esportivos e identificar a } \\
\text { audiência infantil. }\end{array}$ \\
\hline $\begin{array}{l}\text { Youth exposure to alcohol advertising on } \\
\text { television in the UK, the Netherlands and } \\
\text { Germany/Patil et al., } 2014\end{array}$ & Transversal/Nível IV & $\begin{array}{l}\text { Avaliar a exposição dos jovens à publicidade de } \\
\text { álcool veiculada na televisão no Reino Unido, na } \\
\text { Holanda e na Alemanha. }\end{array}$ \\
\hline $\begin{array}{l}\text { The relationship between population-level } \\
\text { exposure to alcohol advertising on television } \\
\text { and brand-specific consumption among } \\
\text { underage youth in the US/Ross et al., } 2015\end{array}$ & Transversal/Nível IV & $\begin{array}{l}\text { Investigar a relação entre a publicidade específica } \\
\text { da marca e o consumo entre os jovens dos } \\
\text { Estados Unidos. }\end{array}$ \\
\hline $\begin{array}{l}\text { The relationship between brand-specific } \\
\text { alcohol advertising on television and brand- } \\
\text { specific consumption among underage } \\
\text { youth/Ross et al., } 2014\end{array}$ & Transversal/Nível IV & $\begin{array}{l}\text { Investigar a relação entre as preferências de } \\
\text { marcas de bebidas alcoólicas e sua exposição. }\end{array}$ \\
\hline $\begin{array}{l}\text { Evidence of underage targeting of alcohol } \\
\text { advertising on television in the United } \\
\text { States: lessons from the Lockyer v. Reynolds } \\
\text { decisions/Ross et al., } 2014\end{array}$ & Longitudinal/Nível III & $\begin{array}{l}\text { Identificar o direcionamento de publicidade de } \\
\text { bebidas alcoólicas para os espectadores entre } 18 \\
\text { e } 20 \text { anos. }\end{array}$ \\
\hline $\begin{array}{l}\text { Do UK television alcohol advertisements } \\
\text { abide by the code of broadcast advertising } \\
\text { rules regarding the portrayal of alcohol?/ } \\
\text { Searle et al., } 2014\end{array}$ & Transversal/Nível IV & $\begin{array}{l}\text { Avaliar a percepção do público sobre a violação da } \\
\text { regulamentação de publicidade do álcool no Reino } \\
\text { Unido. }\end{array}$ \\
\hline $\begin{array}{l}\text { Cued recall of alcohol advertising on } \\
\text { television and underage drinking behavior/ } \\
\text { Tanski et al., } 2015\end{array}$ & Longitudinal/Nível III & $\begin{array}{l}\text { Examinar o alcance da publicidade do álcool na } \\
\text { televisão e seu efeito sobre o consumo entre } \\
\text { menores de idade. }\end{array}$ \\
\hline $\begin{array}{l}\text { Industry Television Ratings for } \\
\text { Violence, Sex, and Substance Use/Gabrielli } \\
\text { et al., } 2016\end{array}$ & Transversal/Nível IV & $\begin{array}{l}\text { Examinar se as diretrizes de televisão têm } \\
\text { discriminado sobre violência, sexo, uso de álcool e } \\
\text { tabagismo na TV. }\end{array}$ \\
\hline $\begin{array}{l}\text { Analysis of Alcohol Industry Submissions against } \\
\text { Marketing Regulation/Martino et al., } 2017\end{array}$ & Transversal/Nível IV & $\begin{array}{l}\text { Examinar as submissões da indústria de álcool } \\
\text { australiana com base na atividade política da indústria. }\end{array}$ \\
\hline
\end{tabular}

TV: televisão; BCAP: Código Advertising Standards Authority of Broadcast

Figura 2 - Caracterização dos artigos internacionais quanto ao título, autores, ano de publicação, tipo de estudo, nível de evidência e objetivos.

\begin{tabular}{|l|l|l|}
\hline Título/Autor/Ano & $\begin{array}{l}\text { Tipo de Estudo/ } \\
\text { Nível de Evidência }\end{array}$ & Objetivos \\
\hline $\begin{array}{l}\text { Propaganda de álcool e associação ao consumo } \\
\text { de cerveja por adolescentes/Faria et al., 2011 }\end{array}$ & Transversal/Nível IV & $\begin{array}{l}\text { Analisar a associação entre a propaganda de } \\
\text { álcool e o consumo de cerveja por adolescentes. }\end{array}$ \\
\hline $\begin{array}{l}\text { Ineficácia da autorregulamentação das } \\
\text { propagandas de bebidas alcoólicas: uma } \\
\text { revisão sistemática da literatura internacional/ } \\
\text { Vendrame, Pinsky, 2011 }\end{array}$ & Longitudinal/Nível III & $\begin{array}{l}\text { Analisar a literatura internacional sobre a } \\
\text { autorregulamentação da publicidade de bebidas } \\
\text { alcoólicas. }\end{array}$ \\
\hline $\begin{array}{l}\text { Uso de álcool entre estudantes de medicina } \\
\text { da Universidade Federal do Maranhão/ } \\
\text { Barbosa et al., 2013 }\end{array}$ & Transversal/Nível IV & $\begin{array}{l}\text { Avaliar o uso de álcool entre estudantes de } \\
\text { Medicina de uma universidade federal. }\end{array}$ \\
\hline $\begin{array}{l}\text { Consumo alcoólico entre universitários (as) } \\
\text { da área da saúde da Universidade Federal do } \\
\text { Rio Grande/RS: subsídios para enfermagem/ } \\
\text { Baumgarten et al., 2012 }\end{array}$ & Transversal/Nível IV & $\begin{array}{l}\text { Conhecer os fatores associados e as } \\
\text { consequências do uso de álcool entre } \\
\text { universitários da área de saúde da Universidade } \\
\text { Federal do Rio Grande do Sul. }\end{array}$ \\
\hline $\begin{array}{l}\text { Uso de álcool entre acadêmicos de farmácia de } \\
\text { uma universidade pública/Cavalcante et al., 2012 }\end{array}$ & Transversal/Nível IV & $\begin{array}{l}\text { Determinar a prevalência do consumo de álcool } \\
\text { entre acadêmicos de Farmácia. }\end{array}$ \\
\hline $\begin{array}{l}\text { Prática de binge alcoólico entre estudantes } \\
\text { universitários/Feijão et al., 2012 }\end{array}$ & Transversal/Nível IV & $\begin{array}{l}\text { Investigar o consumo de bebidas alcoólicas por } \\
\text { universitários, com ênfase na prática de binge. }\end{array}$ \\
\hline $\begin{array}{l}\text { Consumo de álcool entre acadêmicos da } \\
\text { área da saúde: implicações para a prática } \\
\text { profissional/Felipe, Gomes, 2014 }\end{array}$ & Transversal/Nível IV & $\begin{array}{l}\text { Identificar o consumo de álcool entre acadêmicos } \\
\text { da saúde e suas implicações na prática } \\
\text { profissional. }\end{array}$ \\
\hline
\end{tabular}




\begin{tabular}{|l|l|l|}
\hline $\begin{array}{l}\text { Fatores de personalidade como preditores } \\
\text { do consumo de álcool por estudantes } \\
\text { universitários/Natividade et al., 2012 }\end{array}$ & Transversal/Nível IV & $\begin{array}{l}\text { Verificar fatores de personalidade entre pessoas } \\
\text { que consumiram álcool nos últimos três meses. }\end{array}$ \\
\hline $\begin{array}{l}\text { Consumo de bebidas alcoólicas e prática do } \\
\text { binge drinking entre acadêmicos da área da } \\
\text { saúde/Nunes et al., } 2012\end{array}$ & Transversal/Nível IV & $\begin{array}{l}\text { Identificar a prevalência e os fatores associados } \\
\text { ao consumo de álcool e binge entre acadêmicos } \\
\text { de saúde. }\end{array}$ \\
\hline $\begin{array}{l}\text { Representações sociais de bebida } \\
\text { alcoólica para homens universitários/Rosa, } \\
\text { Nascimento, 2015 }\end{array}$ & Transversal/Nível IV & $\begin{array}{l}\text { Descrever aspectos das representações sociais de } \\
\text { bebida alcoólica para universitários. }\end{array}$ \\
\hline $\begin{array}{l}\text { Estudo transversal sobre o uso de risco de } \\
\text { álcool em uma amostra de estudantes de } \\
\text { uma universidade federal brasileira/Silva, } \\
\text { Tucci, 2015 }\end{array}$ & Transversal/Nível IV & $\begin{array}{l}\text { Avaliar o consumo de álcool entre estudantes de } \\
\text { universidade federal vinculada à REUNI. }\end{array}$ \\
\hline $\begin{array}{l}\text { Consumo de bebidas alcoólicas entre } \\
\text { estudantes de Enfermagem/Tavares-Jomar, } \\
\text { Santos-Silva, 2013 }\end{array}$ & Transversal/Nível IV & $\begin{array}{l}\text { Identificar o consumo de álcool entre estudantes } \\
\text { de Enfermagem. }\end{array}$ \\
\hline $\begin{array}{l}\text { Padrão de Consumo de Álcool em Estudantes } \\
\text { Universitários (Calouros) e Diferença entre os } \\
\text { Gêneros/Silva, Tucci, 2016 }\end{array}$ & Transversal/Nível IV & $\begin{array}{l}\text { Avaliar o padrão de consumo de álcool entre } \\
\text { calouros, comparar o consumo entre os gêneros e } \\
\text { identificar a exposição de risco. }\end{array}$ \\
\hline $\begin{array}{l}\text { Uso de álcool e outras drogas entre } \\
\text { universitários do sexo masculino e seus } \\
\text { significados/Dázio et al., 2016 }\end{array}$ & Transversal/Nível IV & $\begin{array}{l}\text { Compreender os significados atribuídos pelos } \\
\text { universitários ao uso de álcool e outras drogas. }\end{array}$ \\
\hline
\end{tabular}

REUNI: Reestruturação e Expansão das Universidades Federais

Figura 3 - Caracterização dos artigos nacionais quanto ao título, autores, ano de publicação, tipo de estudo, nível de evidência e objetivos.

\section{Discussão}

A partir da análise dos resultados, verificou-se que, apesar de não serem encontrados estudos que abordassem diretamente a influência da mídia televisiva no consumo de bebidas alcoólicas e sua relação com o grupo específico de universitários, foi possível notar que a mídia, em geral, possui um poder de persuasão considerável e que, em se tratando da televisão, seu poder é potencializado visto que atinge um maior número de pessoas simultaneamente incluindo grupos vulneráveis. Dessa maneira, a partir dos resultados, emergiram três categorias que tratam dos fatores associados ao consumo de bebidas alcoólicas por universitários, às políticas de restrição publicitária de bebidas alcoólicas e às estratégias de marketing de bebidas alcoólicas.

\section{Fatores determinantes do consumo de bebidas alcoólicas por universitários}

O consumo de bebidas alcoólicas é, historicamente, um comportamento comum da sociedade associado a fatores socioculturais. Estudos trazem que esse consumo fundamenta-se na influência do consumo pela família, pelos amigos, pela mídia, bem como pelo ingresso na faculdade, sendo que este último representa um fator propício para a ingestão de álcool visto que os universitários experimentam um momento de liberdade e autonomia para tomar as decisões sem a supervisão ou orientação dos pais ou responsáveis (2,9-10) $^{2}$

A inserção do jovem no meio universitário torna-o vulnerável a diversos comportamentos de risco característicos do grupo populacional(9). Por se tratar de um momento de autonomia, que nem sempre é acompanhado de responsabilidade, a maioria dos universitários apresenta padrão de consumo de bebidas alcoólicas exagerado, o que pode se associar a problemas frequentes nesse meio como o sexo desprotegido, brigas, direção alcoolizada, acidentes, prejuízos sociais e, inclusive, comprometimento no desempenho acadêmico ${ }^{(10-11)}$. Apesar dos riscos, o consumo tem aumentado pelo fato de serem jovens $\mathrm{e}$ pelo livre comércio(12).

Historicamente, o consumo de álcool tem uma maior relação com a população do sexo masculino, sendo essa associação em quantidade e qualidade. Porém, as mulheres têm apresentado alteração no comportamento frente ao consumo de bebidas alcóolicas, se tornando população-alvo a ser conquistada pelo mercado midiático, pois elas têm aumentado o seu consumo tanto em frequência, quanto em volume ${ }^{(10)}$.

Estudos realizados com acadêmicos abordando a motivação do consumo de álcool apontam que esses jovens bebem com a finalidade de obter diversão, juntamente com os amigos, visto que a percepção que esses indivíduos têm sobre o álcool é de que o seu consumo pode proporcionar, ao sujeito, interação social, alegria, autoestima, relaxamento e prazer, além 
de possuir efeito imediato ${ }^{(4,11,13-15)}$. Ainda, o consumo de bebidas alcoólicas pode estar associado a outras drogas e representa, para os estudantes, uma forma de amenizar o estresse causado pela graduação, o favorecimento de relacionamentos afetivos, a afirmação da liberdade e permeia pelas construções socioculturais de gênero ${ }^{(15)}$.

Outro incentivo ao consumo de álcool é a publicidade, que traz consigo elementos capazes de produzir, nos jovens, a necessidade de obter o que é mostrado de modo que eles consomem o produto ao observar os anúncios na crença de que os comerciais de bebidas alcoólicas reproduzem uma realidade a partir da similaridade entre as festas frequentadas por eles e as expostas nos comerciais ${ }^{(5)}$. Há, ainda, uma associação da propaganda com a preferência de uma marca específica, como apresentam estudos em que foram verificadas que as marcas de bebidas alcoólicas não anunciadas em 20 programas de televisão não foram consumidas, enquanto que as bebidas anunciadas foram consumidas cerca de quatro vezes mais do que habitualmente ${ }^{(16-17)}$.

A prática do binge drinking, uma modalidade de consumo em que o indivíduo ingere uma grande quantidade de álcool em um curto período de tempo, também se apresenta como um determinante no consumo de bebida alcoólica ${ }^{(2,13,18-19)}$. Essa prática foi observada em $32,1 \%$ dos etilistas pesquisados em um estudo que relatava a exposição ao risco de desenvolver problemas relacionados ao consumo dessa substância. Portanto, além da elevada prevalência do consumo de bebidas alcoólicas, a quantidade de álcool consumido é uma preocupação à saúde biopsicossocial dos consumidores $^{(15,20)}$.

\section{Políticas de restrição publicitária de bebidas alcoólicas}

A elaboração de restrições de publicidade de bebidas alcoólicas é de responsabilidade de cada país, sendo possível a restrição de propagandas pelo controle legal por meio das leis, pela autorregulação, por meio do controle estabelecido pela ética da própria empresa, ou por ambas ${ }^{(21)}$. Desse modo, há nações que são mais criteriosas nesse aspecto como, por exemplo, o Reino Unido, a Alemanha e a Holanda, que possuem uma gama de políticas fiscalizatórias relativas ao consumo de bebidas alcoólicas ${ }^{(22)}$.

No intuito de proteger as pessoas mais vulneráveis dos potenciais efeitos de marketing de bebidas alcoólicas, a Austrália utiliza-se da estrutura Alcoohol Beverages Advertising (and Packaging) Code Scheme
( $A B A C)$, que consiste em regulações pautadas nas seguintes fases: autorregulação, pré-habilitação e reclamação ou adjudicação pública. No entanto, as violações dos códigos estabelecidos não são sujeitas às sanções legais ${ }^{(23)}$. Ainda, o país é coberto por códigos de restrição a propagandas, o que inclui algumas restrições referentes à publicidade do álcool, porém, verifica-se que, ainda assim, o público vulnerável é alcançado(23).

No Brasil, vigora a Lei 9.294 , de 15 de julho de 1996, que dispõe sobre a publicidade e a propaganda de bebidas alcoólicas e outros produtos considerando como bebidas alcóolicas aquelas com teor alcoólico superior a treze graus Gay-Lussac ( ${ }^{\circ} \mathrm{GL}$ ) e excluindo, assim, os vinhos e as cervejas ${ }^{(5,21)}$. Dessa maneira, livres das ações fiscalizatórias, as indústrias cervejeiras são as principais a ignorar os prejuízos sociais causados pelo consumo exagerado da cerveja, sendo uma das maiores investidoras em propagandas.

Além da lei supracitada, no Brasil, existe o Conselho Nacional de Autorregulamentação Publicitária (CONAR), que se trata de um órgão não governamental criado por publicitários na tentativa de evitar medidas governamentais de proibição da propaganda utilizando um sistema de controle pautado na ética, a autorregulamentação, apresentada como uma alternativa entre os prejuízos da competição anunciantes/direitos do consumidor versus a delegação regulamentar dos governantes, uma vez que suas ações legislativas nem sempre demonstram compreender a astúcia da publicidade comercial|(5,21).

O CONAR é uma estratégia importante visto que enfatiza que a publicidade não deve alcançar o público infantil/jovem não sendo, também, permitida a indução ao consumo abusivo dos produtos, bem como a associação a imagens sexuais, desempenho saudável e esportes, além de exigir respeito à restrição de horários e à adição de advertências em anúncios ${ }^{(5)}$. No entanto, embora a autorregulamentação tenha o objetivo de reduzir os danos causados pelo etilismo, na prática, ela traz pouca proteção ao público vulnerável devido à frequente violação dos códigos pelas indústrias de álcool que possibilita interpretações subjetivas, atinge públicos ilegais e incentiva o consumo irresponsável, assim como apontam estudos realizados em diferentes nações ${ }^{(16,19,21-22)}$.

Um estudo sobre a percepção da violação de regras de publicidade de bebidas alcoólicas apresenta que $75 \%$ dos participantes identificaram a violação de, pelo menos, uma das dezesseis regras do Código Advertising Standards Authority of Broadcast Publicity (BCAP), código adotado no Reino Unido para evitar anúncios irresponsáveis ${ }^{(24)}$. Outros estudos corroboram a informação e acrescentam que as propagandas de 
bebidas alcoólicas são realmente direcionadas ao público jovem confirmando a ideia de que os regulamentos de publicidade não têm sido efetivos na limitação de jovens à exposição à publicidade de bebida alcoólica na televisão e, consequentemente, a mídia tem influenciado o comportamento dos telespectadores ${ }^{(16,19,22,25)}$.

Ao analisar o posicionamento das empresas de bebidas alcoólicas quanto aos regulamentos de publicidade do produto, bem como às suas atualizações para a proteção ao público vulnerável, em especial, crianças e adolescentes, a indústria mostrou-se resistente sob as seguintes alegações: 1 - O regulamento é redundante; 2 -As evidências de que o marketing de bebidas alcoólicas influencia o aumento do consumo são insuficientes, 3 - O aumento da regulação propicia consequências não intencionais como, por exemplo, a dificuldade da empresa em se manter no comércio e a perda de consumidores; 4 - Inconsistência legal ao considerarem que não foi apresentada uma Declaração de Impacto Regulamentar antes da proposta de revisão da regulamentação e 5 Possuem responsabilidade social visto que se esforçam para reduzir o consumo prejudicial por meio de programas e campanhas conscientes, por exemplo ${ }^{(23)}$.

\section{Estratégias de marketing das empresas de bebidas alcoólicas}

A publicidade das empresas de bebidas alcoólicas busca, a partir de suas propagandas bem elaboradas e aplicadas, influenciar o receptor da mensagem a consumir seus produtos. Acredita-se que uma das maiores estratégias seria aproveitar-se da complexidade das regras e das falhas no controle da autorregulamentação para aumentar o número de vendas e atingir novos consumidores. Assim, os anúncios comerciais de bebidas alcoólicas vêm sendo direcionados, cada vez mais, para o público jovem, acompanhados do incentivo de beber(21).

Estudo realizado com o objetivo de verificar a adequação das transmissões na TV em relação às diretrizes para comportamentos de risco, como violência, comportamento sexual, consumo de bebidas alcoólicas e tabagismo, evidenciou que o álcool esteve presente em $58 \%$ dos programas, em geral, em 7,2 segundos por minuto, $(P<0.001)$, com prevalência inferior apenas às referências para a violência ${ }^{(26)}$.

Embora o consumo seja predominante no sexo masculino, as mulheres estão fazendo uso de bebidas alcoólicas cada vez mais e, possivelmente, alcançarão os homens na prevalência de consumo de bebidas alcoólicas ${ }^{(2,11)}$. A mudança nesse contexto de consumo pode estar relacionada às mudanças na atitude cultural, bem como no empenho da mídia em atingir novo público.

A influência das propagandas de bebidas alcoólicas atinge pessoas de todas as faixas etárias, em especial, os menores de idade, devido ao maior tempo de exposição à mídia televisiva e, consequentemente, ao marketing do produto. Essas estimulam o imaginário do telespectador à criação de uma pseudorrealidade buscando associar o que é exposto pela mídia com o que é vivido pelos indivíduos ${ }^{(5)}$. Nesse sentido, observase a perspectiva da realidade buscada pelos jovens, que acreditam alcançar benefícios por meio do consumo, entre esses, o desejo próprio, o divertimento com os amigos e as celebrações ${ }^{(3-4,14)}$.

Embora as empresas de álcool neguem a promoção de seus produtos para os jovens, é comumente vista, na televisão, a demonstração de tais bebidas em diversos programas populares para a específica faixa etária contribuindo, dessa maneira, para o consumo cada vez mais precoce ${ }^{(27-28)}$. Estudo realizado na Austrália, em 2012, aponta que os telespectadores que assistem à televisão nos horários mais cedo possuem entre cinco e 29 anos de idade, havendo um maior número de crianças de zero a quatro anos. Indivíduos com idades superiores (18 a 29 anos) só foram prevalentes entre $20 \mathrm{~h} 30$ e $23 \mathrm{~h} 59$ e, mesmo nesses horários, o número de telespectadores menores de idade foi superior ${ }^{(28)}$.

Os esforços das empresas de bebidas alcoólicas são incansáveis na busca de lucro, expansão comercial e fidelidade da marca por meio de altos investimentos no marketing de seus produtos e patrocínios generosos aos eventos. Assim, das diversas estratégias utilizadas para atingir seu propósito, destaca-se, também, a associação do álcool com culturas prestigiadas pela população. No Brasil, por exemplo, as bebidas estão relacionadas ao futebol e ao Carnaval, enquanto que, nos Estados Unidos, associa-se à liga de baseball e a campeonatos de basquete, modalidades características no país $\mathbf{s}^{(5,21,28)}$.

Um estudo que teve como objetivo determinar a frequência e a natureza das referências visuais ao álcool em amostra de partidas de EURO 2012 transmitidas no Reino Unido identificou que, em 1487 minutos de transmissão, 1846 referências visuais ao álcool foram identificadas correspondendo a uma média de 1,24 referências visuais por minuto de transmissão e, considerando-se a repercussão mundial do evento, essa estratégia tem potencial de atingir o público global(29).

Nesse intento, fica evidente o jogo de interesse das empresas de bebidas alcoólicas quando estas associam seus produtos com modalidades esportivas, patrocinando esses eventos e expondo o álcool a uma extensa população sem restrição de horário e público, como corrobora outro estudo que relata que 
a distribuição de insinuações de bebidas alcoólicas esteve presente em diversos gêneros e em mais da metade de todas as telenovelas e longas-metragens, destacando-se a proporção de intervalos contendo o uso de álcool significantemente maior em horários de maior audiência no exterior, ou seja, das 6 h às 22 horas ${ }^{(27-28)}$.

\section{Conclusão}

Este estudo evidenciou que as propagandas de bebidas alcoólicas divulgadas na mídia televisiva exercem influência no consumo do produto a partir do uso de elementos que despertam, no jovem, o interesse em vivenciar aquilo que lhe é mostrado como, por exemplo, socialização, diversão, liberdade, mulheres e outros.

Para garantir a lucratividade das empresas de bebidas alcoólicas, as propagandas apresentamse cada vez mais atraentes objetivando conquistar novos consumidores. Nessa perspectiva, diante da boa aceitação das bebidas alcoólicas e da vulnerabilidade dos universitários ao consumo irresponsável, considerase a necessidade de se utilizarem algumas alternativas para, ao menos, proteger a população vulnerável como, por exemplo, educar os jovens de maneira que estes exerçam a sua criticidade na análise do que é exposto pela mídia, bem como supervisionar as políticas de restrição de publicidade.

\section{Referências}

1. World Health Organization-WHO. Global status report on alcohol and health [Internet]. 2011 [cited 2016 Ago 21] Available from: <http://www.who.int/substance_abuse/ publications/global_alcohol_report/msbgsruprofiles.pdf > . 2. Baumgarten LZ, Gomes VLO, Fonseca AD. Consumo alcoólico entre universitários(as) da área da saúde da Universidade Federal do Rio Grande/RS: subsídios para enfermagem. Esc Anna Nery. 2012; 16 (3): 530-35.

3. Natividade JC, Aguirre AR, Hutz LBCS. Fatores de personalidade como preditores do consumo de álcool por estudantes universitários. Cad Saúde Pública. 2012; 28 (6): 1091-100.

4. Rosa LFA, Nascimento ARF. Representações sociais de bebida alcoólica para homens universitários. Arq Bras Psicol. 2015; 67(1):3-19.

5. Faria R, Vendrame A, Silva R, Pinsky I. Propaganda de álcool e associação ao consumo de cerveja por adolescentes. Rev Saúde Pública. 2011;45(3):441-7.

6. Lei $n^{\circ} 9.294$, de 15 de julho de 1996 (BR). Dispõe sobre as restrições ao uso e à propaganda de produtos fumígeros, bebidas alcoólicas, medicações, terapias e defensivos agrícolas [Internet]. Diário Oficial da União. 1996 [Acesso
21 ago 2016]. Acesso em: <http://www.planalto.gov.br/ ccivil_03/leis/L9294.htm>.

7. Mendes KDS, Silveira RCCP, Galvão CM. Revisão integrativa: método de pesquisa para a incorporação de evidências na saúde e na enfermagem. Texto Contexto Enferm. [Internet]. 2008; 17(4):758- 64. Disponível em: <http://www.scielo.br/scielo.php?script=sci_ar ttext\&pid=S0104-07072008000400018\&Ing=en>. Acesso 9 Abr 2018.

8. Minayo MCS. O desafio do conhecimento. 10. ed. São Paulo: Hucitec; 2007.

9. Silva EC, Tucci AM. Estudo transversal sobre o uso de risco de álcool em uma amostra de estudantes de uma universidade federal brasileira. J Bras Psiquiatr. 2014;63(4):317-25.

10. Silva EC, Tucci AM. Padrão de consumo de álcool em estudantes universitários (calouros) e diferença entre os gêneros. Temas Psicol. 2016; 24(1):313-23.

11. Barbosa FL, Barbosa RL, Barbosa MCL, Aguiar DL, Figueiredo IA, Ribeiro AC, et al. Uso de Álcool entre Estudantes de Medicina da Universidade Federal do Maranhão. Rev Bras Educ Médica. 2013;37(1):89-95.

12. Cavalcante DB, Gomes RIB, Sousa VEC, Sardinha AHL, Costa MR Filho. Uso de álcool entre acadêmicos de farmácia de uma universidade pública. Rev Enferm UERJ. 2012;20(3):312-6.

13. Feijão IEP, Sampaio HAC, Sabry MOD, Carioca AAF, Yum MEM, Lima JWO. Prática de binge alcoólico entre estudantes universitários. Rev Bras Promoção Saúde. 2012;25(4):462-8.

14. Felipe ICV, Gomes AMT. Consumo de álcool entre acadêmicos da área da saúde: implicações para a prática profissional. Rev Enferm UERJ. 2014;22(1): 35-41.

15. Dázio EMR, Zago MMF, Fava SMCL. Uso de álcool e outras drogas entre universitários do sexo masculino e seus significados. Rev Esc Enferm USP. 2016; 50(5):786-92. 16. Ross CS, Maple E, Siegel M, DeJong W, Naimi TS, Ostroff J, et al. The Relationship between Brand-Specific Alcohol Advertising on Television and Brand-Specific Consumption among Underage Youth. Alcohol Clin Exp Res. 2014;38(8):2234-42.

17. Ross CS, Maple E, Siegel M, DeJong W, Naimi TS, Padon AA, et al. The Relationship Between PopulationLevel Exposure to Alcohol Advertising on Television and Brand-Specific Consumption Among Underage Youth in the US. Alcohol Alcoholism. 2015;50(3):358-64.

18. Nunes JM, Campolina LR, Vieira MA, Caldeira AP. Consumo de bebidas alcoólicas e prática do binge drinking entre acadêmicos da área da saúde. Rev Psiq Clín. 2012;39(3): 94-9.

19. Tanski SE, McClure AC, Li Z, Jackson K, Morgenstern M, $\mathrm{Li} Z$ et al. Cued Recall of Alcohol Advertising on Television 
and Underage Drinking Behavior. JAMA Pediatr. 2015;169(3):264-71.

20. Tavares-Jomar R, Santos-Silva E. Consumo de bebidas alcoólicas entre estudantes de Enfermagem. Aquichan. 2013;13(2):226-33.

21. Vendrame A, Pinsky I. Ineficácia da autorregulamentação das propagandas de bebidas alcoólicas: uma revisão sistemática da literatura internacional. Rev Bras Psiquiatria. 2011;33(2):196-202.

22. Patil S, Winpenny EM, Elliott MN, Rohr C, Nolte E. Youth exposure to alcohol advertising on television in the UK, the Netherlands and Germany. Eur J Public Health. 2014;24(4):561-5.

23. Dázio EMR, Zago MMF, Fava SMCL. Uso de álcool e outras drogas entre universitários do sexo masculino e seus significados. Rev Esc Enferm USP. 2016; 50(5):786-92.

24. Searle R, Alston D, French DP. Do UK Television Alcohol Advertisements Abide by the Code of Broadcast Advertising Rules Regarding the Portrayal of Alcohol? Alcohol Alcoholism. 2014;49(4):472-8.

25. Ross CS, Ostroff J, Jernigan DH. Evidence of underage targeting of alcohol advertising on television in the United States: Lessons from the Lockyer v. Reynolds decisions. J Public Health Policy. 2014;35(1):105-18.

26. Gabrielli J, Traore A, Stoolmiller M, Bergamini E, Sargent JD. Industry television ratings for violence, sex, and substance use. Pediatrics. 2016;138 (3):e20160487. 27. Lyons A, McNeill A, Britton J. Alcohol imagery on popularly viewed television in the UK. J Public Health. [Internet]. 2013 [cited Sep 14 2016];36(3):426-34. Available from: https://www.ncbi.nlm.nih.gov/pmc/articles/ PMC4181421/.

28. O'Brien KS, Carr S, Ferris J, Room R, Miller P, Livingston $M$, et al. Alcohol Advertising in Sport and Non-Sport TV in Australia, during Children's Viewing Times. J Plos One. 2015;10(8). https://doi.org/10.1371/journal.pone.0134889 29. Adams J, Coleman J, White M. Alcohol marketing in televised international football: frequency analysis. BMC Public Health. 2014; 14: 473. 